PROCEEDINGS OF THE

AMERICAN MATHEMATICAL SOCIETY

Volume 130, Number 11, Pages 3379-3384

S 0002-9939(02)06451-1

Article electronically published on April 11, 2002

\title{
FIRST STABILITY EIGENVALUE CHARACTERIZATION OF CLIFFORD HYPERSURFACES
}

\author{
OSCAR PERDOMO
}

(Communicated by Bennett Chow)

\begin{abstract}
The stability operator of a compact oriented minimal hypersurface $M^{n-1} \subset S^{n}$ is given by $J=-\Delta-\|A\|^{2}-(n-1)$, where $\|A\|$ is the norm of the second fundamental form. Let $\lambda_{1}$ be the first eigenvalue of $J$ and define $\beta=-\lambda_{1}-2(n-1)$. In 1968 Simons proved that $\beta \geq 0$ for any non-equatorial minimal hypersurface $M \subset S^{n}$. In this paper we will show that $\beta=0$ only for Clifford hypersurfaces. For minimal surfaces in $S^{3}$, let $|M|$ denote the area of $M$ and let $g$ denote the genus of $M$. We will prove that $\beta|M| \geq 8 \pi(g-1)$. Moreover, if $M$ is embedded, then we will prove that $\beta \geq \frac{g-1}{g+1}$. If in addition to the embeddeness condition we have that $\beta<1$, then we will prove that $|M| \leq \frac{16 \pi}{1-\beta}$.
\end{abstract}

\section{INTRODUCTION AND PRELIMINARIES}

In 1968, James Simons [S] proved an estimate for the first eigenvalue of the stability operator on any minimal hypersurface $M^{n-1} \subset S^{n}$. In this paper we will show that this estimate is sharp only for the minimal products:

$$
S^{k}\left(\sqrt{\frac{k}{n-1}}\right) \times S^{l}\left(\sqrt{\frac{l}{n-1}}\right) \subset S^{n} \subset \mathbf{R}^{n+1} \quad \text { with } \quad k+l=n-1 .
$$

In the case $k=l=1$ the resulting minimal surface is called the Clifford torus. We will refer to all the products above as Clifford hypersurfaces.

Let $M$ be a compact, oriented minimal hypersurface immersed in the $n$-dimensional sphere $S^{n}$. Let $\nu$ be a unit normal vector field along $M$. For any tangent vector $v \in T_{m} M, m \in M$, the shape operator $A$ is given by $A(v)=-\bar{\nabla}_{v} \nu$, where $\bar{\nabla}$ denotes the Levi Civita connection in $S^{n}$. We will denote by $\Delta$ the Laplacian on $M$. Given any function $f: M \longrightarrow \mathbf{R}^{1}$ we can form the 1-parameter variational family defined by

$$
M_{t}=\{\exp (m, t f(m) \nu): m \in M\}
$$

where $\exp (m, \cdot)$ is the exponential map at $m \in S^{n}$.

Received by the editors September 8, 2000 and, in revised form, June 6, 2001.

2000 Mathematics Subject Classification. Primary 53A10.

(C)2002 American Mathematical Society 
It is well known (see e.g. [SL]) that the $n$-1-dimensional volume of $M_{t}$ satisfies

$$
\begin{aligned}
& \left.\frac{d}{d t}\left(\operatorname{Vol}\left(M_{t}\right)\right)\right|_{t=0}=0 \quad(\text { minimality of } M), \\
& \left.\frac{d^{2}}{d t^{2}}\left(\operatorname{Vol}\left(M_{t}\right)\right)\right|_{t=o}=\int_{M} J(f) f \quad \text { (second variation formula) }
\end{aligned}
$$

where $J$ is the Jacobi or stability operator on $M$, given by

$$
J=-\Delta-\|A\|^{2}-(n-1) .
$$

We will denote the first eigenvalue of $J$ by $\lambda_{1}$. This eigenvalue has the following characterization [C]:

$$
\lambda_{1}=\min \left\{\frac{\int_{M} J(f) f}{\int_{M} f^{2}}: f \in C^{\infty}(M), f \not \equiv 0\right\}
$$

and it is known that its multiplicity is 1 . Let $\rho$ be an eigenfunction of $J$ associated with $\lambda_{1}$.

The easiest minimal hypersurfaces to describe are the equators, i.e. the totally geodesic $S^{n-1}$ 's in $S^{n}$, and the Clifford hypersurfaces defined above.

Because of the symmetries of these minimal hypersurfaces, equators and Clifford hypersurfaces have $\|A\|^{2}$ constant. Therefore, the stability operator and the laplacian differ by a constant, hence, it is not difficult to show that $\lambda_{1}=-(n-1)$ for the equators and $\lambda_{1}=-2(n-1)$ for the Clifford hypersurfaces.

In this paper we will show that the only minimal hypersurfaces with $\lambda_{1}=$ $-2(n-1)$ are the Clifford hypersurfaces. For minimal surfaces in $S^{3}$, we will give an additional identity that relates the genus $g$ of $M$, the area $|M|$ of $M, \lambda_{1}$, and the simple invariant $\alpha=\int_{M} \frac{\|\nabla \rho\|^{2}}{\rho^{2}}$. Notice that this invariant is independent of the choice of $\rho$ because the multiplicity of $\lambda_{1}$ is 1 . We also have that $\alpha$ is defined not only for surfaces but for any minimal hypersurface in $S^{n}$ and that $\alpha=0$ if and only if $\|A\|$ is constant.

In [S] Simons studied the function $\|A\|$ and he deduced that if $M$ is not an equator, then $\lambda_{1} \leq-2(n-1)$. This result allowed him to deduce that the only stable cones in $\mathbf{R}^{n}, n \leq 7$, are the ones that come from equators, i.e. hyperplanes. The result we just mentioned and the main result in this paper use the following elliptic equation for the shape operator, $A$, found by Simons [S]:

$$
\Delta A=(n-1) A-\|A\|^{2} A .
$$

The following theorem, proven by Chern, DoCarmo and Kobayashi [C-D-K] and independently by Lawson [L1], gives another consequence of this elliptic equation:

Theorem 1.2. If $M$ is a compact orientable minimal hypersurface on $S^{n}$ with $\|A\|^{2} \equiv n-1$, then $M$ is a Clifford hypersurface.

In section $\S 2$ we find an elliptic inequality for the function $f=\|A\| \rho^{-1}$, that will help us, after applying the maximum principle, to deduce that $\lambda_{1}=-2(n-1)$ implies that $\|A\|$ is a first eigenfunction of the stability operator.

In section $\S 3$ we compute the laplacian of the function $h=\ln (\rho)$, then we deduce, after applying Stokes' theorem, the identity for minimal surfaces we mentioned earlier. 


\section{2. $\lambda_{1}$-CHARACTERIZATION OF ClifFORD HYPERSURFACES}

In this section we characterize the Clifford hypersurfaces as the only minimal immersions whose first stability eigenvalue, $\lambda_{1}$, equals $-2(n-1)$.

Before we state and prove our main theorem of this section we will make some computations. Choose a first eigenfunction, $\rho$, of the stability operator with $\rho>0$. Then we have

$$
-\Delta \rho-\|A\|^{2} \rho-(n-1) \rho=\lambda_{1} \rho .
$$

For any $v, w \in T_{m} M$, denote by $D_{v} A(w)$ the covariant tensor derivative of the shape operator $A$. Using that $\Delta A=(n-1) A-\|A\|^{2} A$ (equation (1.1)), we obtain, assuming $\|A\|(m) \neq 0$,

$$
\begin{aligned}
\Delta\|A\| & =\operatorname{div}(\nabla\|A\|) \\
& =\operatorname{div}\left(\frac{1}{2}\|A\|^{-1} \nabla\|A\|^{2}\right) \\
& =\frac{1}{2}\left(\left\langle\nabla\|A\|^{-1}, \nabla\|A\|^{2}\right\rangle+\|A\|^{-1} \Delta\langle A, A\rangle\right) \\
& =-\|A\|^{-1} \mid \nabla\|A\|^{2}+\|A\|^{-1}\left(\langle\Delta A, A\rangle+|D A|^{2}\right) \\
& =(n-1)\|A\|-\|A\|^{3}+\|A\|^{-3}\left(\|A\|^{2}\langle D A, D A\rangle-\|A\|^{2} \mid \nabla\|A\|^{2}\right) .
\end{aligned}
$$

Taking an orthonormal basis $\left\{e_{1}, \ldots, e_{n-1}\right\}$ of $T_{m} M$ we have

$$
\begin{aligned}
\left(\|A\|^{2}\langle D A, D A\rangle-\|A\|^{2}|\nabla\|A\||^{2}\right) & =\|A\|^{2} \sum_{i=1}^{n-1}\left\langle D_{e_{i}} A, D_{e_{i}} A\right\rangle-\frac{1}{4}\left\langle\nabla\|A\|^{2}, \nabla\|A\|^{2}\right\rangle \\
& =\|A\|^{2} \sum_{i=1}^{n-1}\left\langle D_{e_{i}} A, D_{e_{i}} A\right\rangle-\frac{1}{4} \sum_{i=1}^{n-1}\left(e_{i}\|A\|^{2}\right)^{2} \\
& =\|A\|^{2} \sum_{i=1}^{n-1}\left\langle D_{e_{i}} A, D_{e_{i}} A\right\rangle-\sum_{i=1}^{n-1}\left\langle A, D_{e_{i}} A\right\rangle^{2}
\end{aligned}
$$

Therefore using the Cauchy-Schwarz inequality we get

Lemma 2.1. $\Delta\|A\| \geq(n-1)\|A\|-\|A\|^{3}$ and equality holds if and only for any vector $v \in T_{m} M D_{v} A=\beta(v) A$, for some linear function $\beta$ on $T_{m} M$.

Define $f=\|A\| \rho^{-1}$. Let $f\left(m_{0}\right)$ be the maximum of $f$ and let $\Omega$ be a region around $m_{0}$ in which $f$ is greater than some positive constant. $\Omega$,

$$
\begin{aligned}
\Delta f=\Delta\left(\rho^{-1}\|A\|\right) & =\|A\| \Delta \rho^{-1}+2\left\langle\nabla \rho^{-1}, \nabla\|A\|\right\rangle+\rho^{-1} \Delta\|A\| \\
& \geq\|A\|\left(2 \rho^{-3}|\nabla \rho|^{2}-(n-1) \rho^{-1}+\|A\|^{2} \rho^{-1}\right) \\
& +\rho^{-1}\left((n-1)\|A\|-\|A\|^{3}\right)+2\left\langle\nabla \rho^{-1}, \nabla\|A\|\right\rangle \\
& =2 \rho^{-3}|\nabla \rho|^{2}\|A\|-2 \rho^{-2}\langle\nabla \rho, \nabla\|A\|\rangle \\
& =-2 \rho^{-1}\left\langle\nabla\left(\rho^{-1}\|A\|\right), \nabla \rho\right\rangle=-2 \rho^{-1}\langle\nabla f, \nabla \rho\rangle .
\end{aligned}
$$

This puts us in the position to prove:

Theorem 2.3. If $M \subset S^{n}$ is a compact oriented immersed minimal hypersurface with $\lambda_{1}=-2(n-1)$, then $M$ is a Clifford hypersurface. 
Proof. Assume $\lambda_{1}=-2(n-1)$. Then $M$ is not totally geodesic, and therefore the function $f=\rho^{-1}\|A\|$ reaches a positive maximum. Letting $\Omega$ be defined as above, we have by $(2.2)$ that

$$
\Delta f+2 \rho^{-1}\langle\nabla f, \nabla \rho\rangle \geq 0 \quad \text { on } \quad \Omega .
$$

Since the maximum of $f$ in $\Omega$ is obtained in the interior of $\Omega$ we get by the maximum principle that $f$ is constant in $\Omega$. Since $M$ is connected, we deduce that $f$ is constant in all $M$, i.e $\|A\|=c \rho$ is itself a first eigenfunction of the stability operator. Now, since $\lambda_{1}=-2(n-1)$, we get $\Delta\|A\|=(n-1)\|A\|-\|A\|^{3}$, hence Lemma 2.1 gives us a 1 -form $\beta$ on $M$ such that

$$
D_{v} A=\beta(v) A .
$$

We now prove that $A$ is parallel. Fix $m \in M$ and choose $\left\{e_{1}, \ldots, e_{n-1}\right\} \in T_{m} M$ an orthonormal basis that diagonalizes $A$. Then the Codazzi equations in $S^{n}$ give us

$$
\left\langle\sum_{i=1}^{n-1} D_{e_{i}} A\left(e_{k}\right), e_{i}\right\rangle=\left\langle\sum_{i=1}^{n-1} D_{e_{k}} A\left(e_{i}\right), e_{i}\right\rangle=\beta\left(e_{k}\right) \sum_{i=1}^{n-1}\left\langle A\left(e_{i}\right), e_{i}\right\rangle=0 .
$$

On the other hand, using (2.4) we conclude

$$
\left\langle\sum_{i=1}^{n-1} D_{e_{i}} A\left(e_{k}\right), e_{i}\right\rangle=\sum_{i=1}^{n-1}\left\langle\beta\left(e_{i}\right) A\left(e_{k}\right), e_{i}\right\rangle=\beta\left(e_{k}\right)\left\langle A\left(e_{k}\right), e_{k}\right\rangle .
$$

If $\left\langle A\left(e_{k}\right), e_{k}\right\rangle \neq 0$, then $\beta\left(e_{k}\right)=0$ and $D_{e_{k}} A=0$. If $\left\langle A\left(e_{k}\right), e_{k}\right\rangle=0$, then for any $w \in T_{m} M$,

$$
D_{e_{k}} A(w)=D_{w} A\left(e_{k}\right)=\beta(w) A\left(e_{k}\right)=0
$$

and we get again that $D_{e_{k}} A=0$.

Since this holds for all $k, A$ is parallel. It follows that $\|A\|$ is constant. The stability equation then shows $\|A\|^{2}=n-1$. Theorem 1.2 then implies the result.

Remark 2.5. For $n=7$ we showed that $\lambda_{1}<-12$ if $M$ is not an equator or a Clifford hypersurface. An improvement of the previous estimate to $\lambda_{1} \leq-12.25$ would be of great interest, as it would show that the only stable cones in $\mathbf{R}^{8}$ are hyperplanes (cones over equators) and cones over Clifford hypersurfaces in $S^{7}[\mathrm{~S}$ ]. Together with the results in [SS], this would yield a complete classification of all area-minimizing hypersurfaces in $\mathbf{R}^{8}$.

\section{Minimal surfaces in $S^{3}$}

In this section, for the case $n=3$, i.e. for $M$ an oriented minimal immersed surface of $S^{3}$, we will find an identity relating the genus of the surface, its area, the value $\alpha$ defined in $\S 1$, and $\lambda_{1}$. This identity will give us a different proof of the result in $\S 2$ and of Simons' result in [S] which states that $\lambda_{1} \leq-4$ if $M$ is not an equator.

Let $\rho$ be as in $\S 2$, we have

$$
-\Delta \rho-\|A\|^{2} \rho-(n-1) \rho=\lambda_{1} \rho .
$$


Let us compute $\Delta \ln \rho$ :

$$
\begin{aligned}
\Delta \ln \rho & =\operatorname{div}(\nabla(\ln \rho)) \\
& =\operatorname{div}\left(\rho^{-1} \nabla(\rho)\right) \\
& =\left\{\left\langle\nabla \rho^{-1}, \nabla \rho\right\rangle+\rho^{-1} \Delta \rho\right\} \\
& =\left\{(-1) \rho^{-2}|\nabla \rho|^{2}+\left(-\lambda_{1}-\|A\|^{2}-(n-1)\right)\right\} .
\end{aligned}
$$

Integrating the equation above we find

$$
\int_{M} \rho^{-2}|\nabla \rho|^{2}=\left(-\lambda_{1}-(n-1)\right)|M|-\int_{M}\|A\|^{2} .
$$

In the case where $M$ is a minimal surface, the Gauss equation, gives us a relation between the norm of the shape operator, $\|A\|^{2}$, and the Gauss curvature of the surface, $K$. Namely,

$$
K=1-\frac{\|A\|^{2}}{2}
$$

If we integrate the relation above and use Gauss-Bonnet, we get

$$
8 \pi(1-g)=2|M|-\int_{M}\|A\|^{2} .
$$

Now combining the equation above with (3.1) we obtain the following proposition.

Proposition 3.2. Let $M$ be a compact oriented minimal immersed in $S^{3}$. If $\rho$ is an eigenfunction associated to the first eigenvalue of the stability operator $\lambda_{1}$ and we define $\alpha=\int_{M} \frac{\|\nabla \rho\|^{2}}{\rho^{2}}$, then

$$
\alpha+8 \pi(g-1)=\left(-\lambda_{1}-4\right)|M| .
$$

Corollary 3.3. Let $M$ be a compact non-totally geodesic oriented minimal surface in $S^{3}$. Then the first eigenvalue of the stability operator, $\lambda_{1}$, satisfies $\lambda_{1} \leq-4$. Moreover, $\lambda_{1}=-4$ if and only if $M$ is a Clifford torus.

Proof. Since $M$ is non-totally geodesic, then the genus, $g$, of $M$ is greater than 0 , because the equator is the only minimal immersion of a sphere in $S^{3}$, [A]. Now, since $g \geq 1$, we get from the proposition above that $\lambda_{1} \leq-4$, with equality only if $g=1$ and $\rho$ is a constant function. The stability equation gives us that if $\rho$ is constant and $\lambda_{1}=-4$, then $\|A\|^{2} \equiv 2$. Therefore $M$ is a Clifford torus by Theorem 1.2 .

Remark 3.4. If we drop $\alpha$ in Proposition 3.2 and we define $\beta=\left(-\lambda_{1}-4\right)$, then we get

$$
8 \pi(g-1) \leq \beta|M|
$$

This inequality can also be achieved by plugging the test function $f \equiv 1$ into the Rayleigh-quotient (see characterization of $\lambda_{1}$ in the introduction). If $M$ is embedded, then Choi and Wang [C-W] proved that $|M| \leq 8 \pi(g+1)$. Combining this inequality with (3.5) above we get

$$
\beta \geq \frac{g-1}{g+1} \quad \text { and if } \beta<1 \text {, then } \quad|M| \leq \frac{16 \pi}{1-\beta} .
$$


Moreover, if $M$ is embedded by the first eigenfuntions of the laplacian, i.e. if Yau's conjecture were true, then Yang and Yau $[\mathrm{Y}-\mathrm{Y}]$ proved that $|M| \leq 4 \pi(g+1)$, therefore the inequalities above can be improved to

$$
\beta \geq 2 \frac{g-1}{g+1} \quad \text { and if } \beta<2, \text { then } \quad|M| \leq \frac{16 \pi}{2-\beta} .
$$

\section{ACKNOWLEDGEMENTS}

I would like to thank my advisor, Professor Bruce Solomon, for his lessons on mathematics and for his supervision that guided me to the understanding and achievement of this paper which is part of my Ph.D. dissertation $[\mathrm{P}$. I would also like to thank Professor Matthew Gursky for showing me the right function to study in the main theorem of this paper, and the referee of this paper for his comments and for pointing out the results in Remark 3.4.

\section{REFERENCES}

[A] F. Almgren, Some interior regularity theorems for minimal surfaces and an extension of Bernstein theorem, Ann. of Math. 85 (1966) pp 277-292. MR 34:702

[C] Isaac Chavel, Eigenvalues in Riemannian Geometry, Pure and Applied Mathematics. 115, Academic Press, 1984. MR 86g:58140

[C-D-K] S.S. Chern, M. DoCarmo, and S. Kobayashi, Minimal submanifolds of a sphere with second fundamental form of constant length, Functional Analysis and Related Fields, Proc. Conf. M. Stone, Springer, 1970, pp 59-75. MR 42:8424

[C-W] H. Choi and A. Wang, A first eigenvalue estimate for minimal hypersurfaces, J. Differential Geometry 18 (1983) pp 559-562. MR 85d:53028

[L] H. B. Lawson, Complete minimal surfaces in $S^{3}$, Ann. of Math. (2) 92 (1970) pp 335374. MR 42:5170

[L1] H. B. Lawson, Local rigidity theorems for minimal hypersurfaces, Ann. of Math. (2) 89 (1969) pp 187-197. MR 38:6505

[P] O. Perdomo, First eigenvalue and index: Two characterizations of minimal Clifford hypersurfaces of spheres, Ph.D. Thesis, Indiana University, 2000.

[S] J. Simons, Minimal Varieties in Riemannian manifolds, Ann. of Math. 88 (1968), pp 62-105.

[SL] L. Simon, Lectures on Geometry Measure Theory, Proceedings of the Centre for Mathematical Analysis, Australian National University, 3, 1983. MR 87a:49001

[SS] L. Simon, B. Solomon, Minimal hypersurfaces asymptotic to quadratic cones in $\mathbf{R}^{n+1}$, Invent. Math. 86 (1986), pp 535-551. MR 87k:49047

[Y-Y] P. Yang and S.T. Yau, Eigenvalues of the Laplacian of compact Riemann surfaces and minimal submanifolds, Ann. Scuola Norm. Sup. Pisa 7 (1980) pp 55-63. MR 81m:58084

Departamento de Matematicas, Universidad del Valle, Cali, Colombia

E-mail address: osperdom@mafalda.univalle.edu.co 